\title{
Rebleeding From a Vertebral Artery Dissecting Aneurysm After Endovascular Internal Trapping: Adverse Effect of Intrathecal Urokinase Injection or Incomplete Occlusion?
}

\author{
Kenji SugIu, Koji TOKunaGa, Shigeki OnO, \\ Ayumi NISHIDA, and Isao DATE \\ Department of Neurological Surgery, Okayama University Graduate School of Medicine, \\ Dentistry and Pharmaceutical Sciences, Okayama, Okayama
}

\begin{abstract}
A 67-year-old woman suffered rebleeding from a ruptured vertebral artery dissecting aneurysm after endovascular internal trapping. The dissecting aneurysm was initially successfully occluded with the affected vertebral artery using detachable coils. However, rebleeding from the aneurysm occurred on the next day. The rebleeding may have resulted from the thrombolytic effect of urokinase, which was injected intrathecally 3 hours before rebleeding occurred, or the relatively loose coil packing of the aneurysm. This case indicates the potential risk of intrathecal use of thrombolytic agents and the importance of complete tight coil packing of the whole dissected site in the treatment of ruptured vertebral artery dissecting aneurysms.
\end{abstract}

Key words: coil, endovascular treatment, internal trapping, rebleeding, thrombolytic agent

Received February 10, 2009; Accepted April 28, 2009 


\section{Introduction}

Intracranial vertebral artery dissecting aneurysms (VADAs) have recently been recognized as an important cause of subarachnoid hemorrhage (SAH). ${ }^{5,15)}$ The rebleeding rate in the acute stage following $\mathrm{SAH}$ is very high, so ultra-early intervention is strongly recommended in the treatment of ruptured VADAs. ${ }^{5,7,10,13,14)}$ The efficacy and safety of endovascular treatment for ruptured VADAs in the acute stage following $\mathrm{SAH}$ is now widely recognized. $6,9,10,13,14,16)$ Endovascular neurosurgeons initially treated ruptured VADAs by occluding the parent artery at or just proximal to the dissecting site using balloons or fibered platinum coils. ${ }^{1)}$ Such proximal occlusion of the vertebral artery (VA) is similar to surgical clipping of the proximal vertebral artery, which carries the potential risk of rebleeding. ${ }^{4}$ Since the introduction of the Guglielmi detachable coil (GDC), endovascular treatment has become safer and more effective because the detachable coil is soft and more easily controlled. ${ }^{13,14,16)}$ The emphasis of endovascular treatment then shifted from proximal occlusion to internal trapping, ${ }^{2,13,14,16)}$ in which the dissected site is completely occluded with detachable coils. This procedure can completely exclude the aneurysm from the circulation and theoretically carries no risk of rebleeding. ${ }^{9)}$

We report a case of rebleeding after endovascular internal trapping of the ruptured VADA and discuss the mechanism of rebleeding.

\section{Case Report}

A 67-year-old woman was admitted to our hospital 4 hours after sudden onset of headache. Computed tomography (CT) revealed SAH. Emergent digital subtraction angiography showed a right VADA (Fig. 1 left).

A 6-Fr guiding catheter was introduced into the right VA with the patient under general anesthesia immediately after diagnostic angiography. An Excel-14 microcatheter (Boston Scientific, Fremont, Calif., U.S.A.) was navigated coaxially into the VADA. Nine GDCs (Boston Scientific) were placed in the aneurysm, which resulted in successful occlusion of the aneurysm with the affected VA. Postoperative angiography revealed complete occlusion of the right VA proximal to the aneurysm and good collateral flow to the posterior circulation through the left VA without retrograde flow into the aneurysm (Fig. 1 right). Lumbar drainage was then placed, and she showed good recovery from general anesthesia. Urokinase $6000 \mathrm{U}$ was injected though the lumbar drainage catheter to wash the subarachnoid clot during the following morning (15 hours after initial onset), because CT showed massive SAH (Fisher group III). She suddenly complained of severe headache 3 hours after the injection of urokinase, and became comatose soon after. Emergent CT demonstrated repeated SAH.

With the patient under general anesthesia, emergent angiography showed complete occlusion of the right VA, and left vertebral angiography demonstrated extravasation of contrast medium at a site distal to the aneurysm (Fig. 2). A
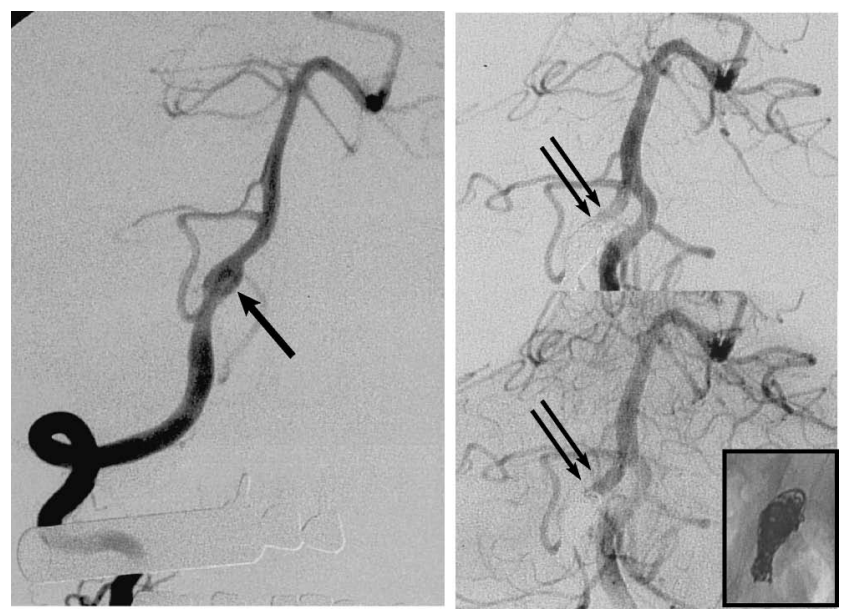

Fig. 1 Left: Initial right vertebral angiogram showing a nonposterior inferior cerebellar artery type vertebral artery dissecting aneurysm with typical "pearl" sign (arrow) at the dissected site. Right: Left vertebral angiograms after the initial procedure showing complete occlusion of the right vertebral artery dissecting aneurysm, which was not opacified (double arrows) despite retrograde flow to the distal part of the right vertebral artery. Upper: early arterial phase, lower: late arterial phase, lower right: shape of coils in the aneurysm.
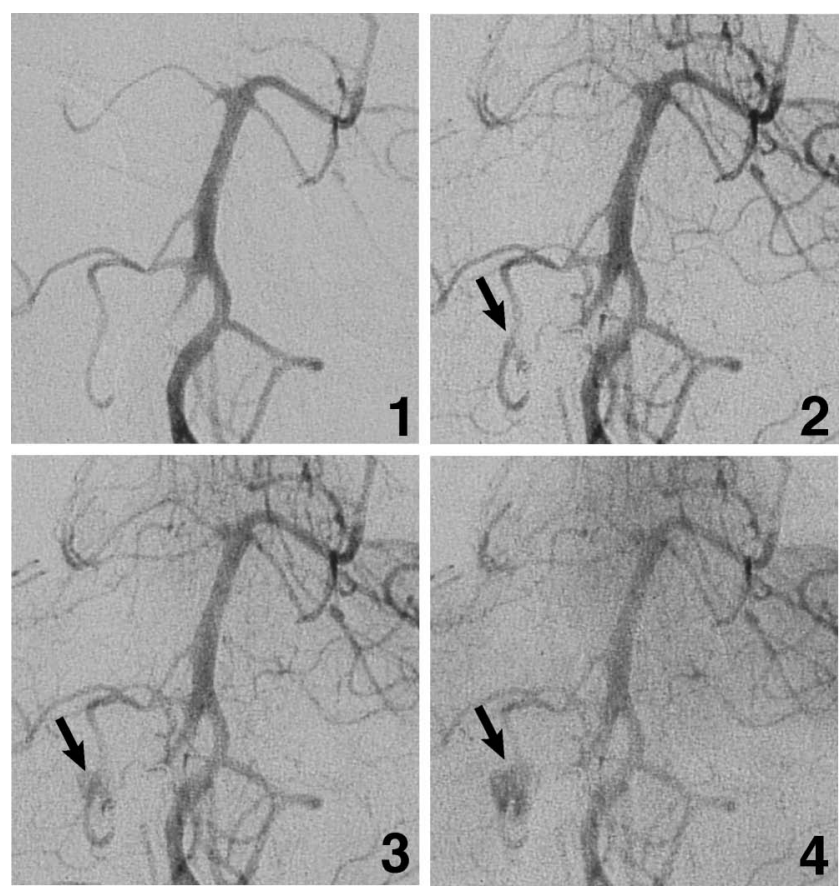

Fig. 2 Serial (1-4) anteroposterior left vertebral angiograms, arterial phase, shortly after rebleeding showing extravasation of contrast medium (arrow) via the retrograde flow into the distal right vertebral artery.

6-Fr guiding catheter was introduced into the left VA. A ProGreat microcatheter (Terumo, Odawara, Kanagawa) was navigated coaxially through the left VA into the distal 


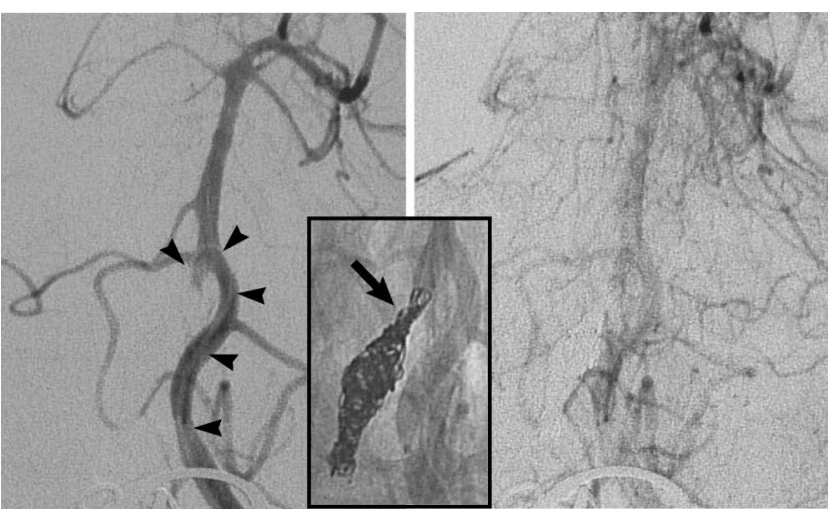

Fig. 3 Left vertebral angiograms after the second embolization showing complete occlusion of the right vertebral artery dissecting aneurysm. Left: Early arterial phase. A microcatheter was navigated through the left vertebral artery into the distal part of the aneurysm in a retrograde fashion (arrowheads). Right: Late arterial phase. Lower center: Shape of coils in the aneurysm. Note the added coils in the distal part of the aneurysm and distal right vertebral artery (arrow).

part of the aneurysm in the retrograde direction. Four GDCs were tightly placed in the aneurysm and the distal part of the right VA, which resulted in complete exclusion of the aneurysm from the circulation (Fig. 3). The patient's neurological condition improved gradually after the second procedure. She was transferred to another hospital to continue rehabilitation 2 months after onset. Severe tetraparesis persisted at the time of discharge.

\section{Discussion}

The typical angiographical finding of VADAs is the "pearl-and-string" sign. ${ }^{5,7)}$ Histological analysis has clearly indicated that the rupture point of VADAs is the dilated part, which is called the "pearl."8,11) Entry-exit cerebral dissecting aneurysms are historically well known as a common type of cerebral dissection, but the majority of ruptured VADAs have entry-only dissection, of which the pseudolumen is the cul-de-sac and rebleeding can easily occur during the acute stage. ${ }^{8)}$ Complete coil packing of the whole dilated part including the affected VA (internal trapping) is therefore recommended as an endovascular procedure to prevent rebleeding in the acute stage following SAH. ${ }^{2,6,9,10,13,14,16)}$

We have treated 60 patients with ruptured VADAs using endovascular techniques between 2000 and 2008..$^{3,13,14)}$ Forty-nine of the 60 patients were treated within 24 hours, because rebleeding is well known to occur in the ultra-early stage of initial rupture. All except two patients were treated by internal trapping using detachable coils. The present case was the only one complicated with rebleeding from the ruptured VADA after endovascular internal trapping. We suspect that relatively loose packing of the distal part of the "pearl" aneurysm, from which extravasation of the contrast medium was seen at second angiography, may have caused the rebleeding. The packing density in the aneurysm in the present case was not partic- ularly dense but was judged to be adequate to prevent rebleeding. We treated several cases with looser coil packing, but no rebleeding occurred in any of these other cases. However, the present case suggests that coil packing of the aneurysm must be as dense as possible to prevent rebleeding. Recanalization of the VADA may occur after internal trapping, which emphasizes the importance of dense packing of the coils during endovascular treatment for VADA. ${ }^{3,12)}$

Intrathecal injection of urokinase was certainly a more important issue. Rebleeding occurred 3 hours after urokinase injection. This was the first and only case in which we injected urokinase intrathecally in our series of 60 cases of ruptured VADAs. We strongly suspect that the thrombolytic effect of the urokinase might have been responsible for the rebleeding, because the arterial wall in VADA is histologically very thin. Interestingly, in a similar case, the patient died of rebleeding one week after proximal occlusion of a VADA, and tissue plasminogen activator was used to clear the patient's ventriculostomy catheter, although whether this injection was related to rebleeding remained unclear. ${ }^{10}$ ) These two cases demonstrate the potential risk of intrathecal use of thrombolytic agents during the treatment of ruptured VADA even if endovascular occlusion has been achieved. We have not used thrombolytic agents to clear cerebrospinal fluid in subsequent cases. We would like to emphasize that intrathecal injection of thrombolytic agents should not be given if only incomplete coil packing was achieved in the endovascular treatment for ruptured VADA.

\section{References}

1) Halbach VV, Higashida RT, Dowd CF, Fraser KW, Smith TP, Teitelbaum GP, Wilson CB, Hieshima GB: Endovascular treatment of vertebral artery dissections and pseudoaneurysms. J Neurosurg 79: 183-191, 1993

2) Iihara K, Sakai N, Murao K, Sakai H, Higashi T, Kogure S, Takahashi JC, Nagata I: Dissecting aneurysms of the vertebral artery: a management strategy. J Neurosurg 97: 259-267, 2002

3) Kikuchi Y, Sugiu K, Tokunaga K, Nishida A, Nishimura T, Date I: [Case of a ruptured vertebral artery dissecting aneurysm recanalized after internal trapping]. No Shinkei Geka 35: 813-819, 2007 (Jpn, with Eng abstract)

4) Kitanaka C, Morimoto T, Sasaki T, Takakura K: Rebleeding from vertebral artery dissection after proximal clipping. Case report. J Neurosurg 77: 466-468, 1992

5) Kitanaka C, Sasaki T, Eguchi T: Intracranial vertebral artery dissections: clinical, radiological features, and surgical considerations. Neurosurgery 34: 620-627, 1994

6) Kurata A, Ohmoto T, Miyasaka Y, Fujii K, Kan S, Kitahara T: Coil embolization for the treatment of ruptured dissecting vertebral aneurysms. AJNR Am J Neuroradiol 22: 11-18, 2001

7) Mizutani T, Aruga T, Kirino T, Miki Y, Saito I, Tsuchida T: Recurrent subarachnoid hemorrhage from untreated ruptured vertebrobasilar dissecting aneurysms. Neurosurgery 36: 905-913, 1995

8) Mizutani T, Kojima H, Asamoto S: Pathological mechanism and three-dimensional structure of cerebral dissecting aneurysms. J Neurosurg 94: 712-717, 2001 
9) Peluso JP, van Rooij WJ, Sluzewski M, Beute GN, Majoie CB: Endovascular treatment of symptomatic intradural vertebral dissecting aneurysms. AJNR Am J Neuroradiol 29: 102-106, 2008

10) Rabinov JD, Hellinger FR, Morris PP, Ogilvy CS, Putman CM: Endovascular management of vertebrobasilar dissecting aneurysms. AJNR Am J Neuroradiol 24: 1421-1428, 2003

11) Sasaki O, Ogawa H, Koike T: A clinicopathological study of dissecting enurysms of the intracranial vertebral artery. $J$ Neurosurg 75: 874-882, 1991

12) Sawada M, Kaku Y, Yoshimura S, Kawaguchi M, Matsuhisa T, Hirata T, Iwama T: Antegrade recanalization of a completely embolized vertebral artery after endovascular treatment of a ruptured intracranial dissecting aneurysm. Report of two cases. J Neurosurg 102: 161-166, 2005

13) Sugiu K, Tokunaga K, Date I: [Endovascular treatment for ruptured vertebral artery dissecting aneurysms]. No Shinkei Geka 32: 1229-1238, 2004 (Jpn)

14) Sugiu K, Tokunaga $K$, Watanabe $K$, Sasahara W, Ono S,
Tamiya T, Date I: Emergent endovascular treatment of ruptured vertebral artery dissecting aneurysms. Neuroradiology 47: 158-164, 2005

15) Yamaura A, Watanabe Y, Saeki N: Dissecting aneurysms of the intracranial vertebral artery. J Neurosurg 72: 183-188, 1990

16) Yamaura I, Tani E, Yokota M, Nakano A, Fukami N, Kaba K, Matsumoto T: Endovascular treatment of ruptured dissecting aneurysms aimed at occlusion of the dissected site by using Guglielmi detachable coils. J Neurosurg 90: 853-856, 1999

Address reprint requests to: Kenji Sugiu, M.D., Department of Neurological Surgery, Okayama University Graduate School of Medicine, Dentistry and Pharmaceutical Sciences, 2-5-1 Shikata-cho, Okayama 700-8558, Japan. e-mail: ksugiu@md.okayama-u.ac.jp 